\title{
A case of temporary correction of drooping nose due to postoperative descent of silicone implant using a filler injection
}

\author{
Kyoungjin Kang, MD, $\mathrm{PhD}^{1}$ (iD, Moon Hee Kim, MD, $\mathrm{MS}^{2}$ (iD, Hyeon Seob Byeon, $\mathrm{MD}^{3}$ (iD \\ ${ }^{1}$ Seoul Cosmetic Surgery Clinic, Busan, Rep. of Korea, ${ }^{2}$ Department of Cosmetic Medicine, GC iMED, Seoul, Rep. of Korea, ${ }^{3}$ Zen Clinic, Zen \\ Medical Corporation, Tokyo, Japan
}

Drooping of the nasal tip is one of the main complications of rhinoplasty. Herein we describe the case of a 42-year-old female who underwent simple augmentation rhinoplasty using an I-shaped silicone implant approximately 3 years ago visited our clinic for the correction of a drooping nose. The contour of the I-shaped silicone was observed in the dorsum of the nasal skin above the supra-tip break point, which caused the nasal tip to droop. The patient refused revision rhinoplasty and requested to temporarily correct the drooping nose using a noninvasive procedure. Sequential augmentation of filler injection (SAFI) for rotation, projection, and augmentation was recommended to correct the condition. In total, $0.6 \mathrm{ml}$ of hyaluronic acid (HA) filler was first injected into the dead space between the anterior nasal spine and end of the medial crus of the lower lateral cartilage and columella for tip rotation. It was then placed intranasally on top of the middle crus of the lower lateral cartilage for tip projection $(0.2 \mathrm{ml})$. Finally, it was injected into the upper lateral cartilage around the supra-tip break for dorsal augmentation $(0.4 \mathrm{ml})$. A total of $1.2 \mathrm{ml}$ of filler was injected. After 2 weeks, $0.2 \mathrm{ml}$ of HA filler was injected for tip rotation. After 4 months, a postoperative follow-up study was performed. The nasolabial angle had changed from $90^{\circ}$ to $105^{\circ}$. Tip projection was significantly increased. The abnormal contour of the silicone implant in the dorsum was no longer visible. The drooping nose remarkably improved with the sequential combination of rotation, projection, and augmentation using the SAFI technique, which considers the dynamic structure of the nasal tip. We were able to demonstrate that the SAFI technique can be a reliable technique for filler rhinoplasty.

Keywords: drooping nose; filler injection for nose; projection; rhinoplasty; rotation

\section{Introduction}

Drooping nose is caused by a variety of factors [1], including intrinsic factors, such as congenitally deformed lower lateral cartilages (LLC), and extrinsic factors, such as disproportionate formation of cartilage and soft tissues, habitual manual pushing of the nasal tip and contraction of the depressor nasi septi muscle, loss of maxillary bone or nasal spine, aging, and previ- ous nasal procedures.

A drooping nose is generally corrected via revision rhinoplasty, which strengthens the tip that supports structure and increases tip rotation and projection [2]. However, some patients do not want surgical management or immediate correction because of the following reasons: fear of reoperation with additional complications, financial restraints, and long downtime. In the present case, the drooping nose was caused by the 
downward migration of an I-shaped silicone implant that was inserted for nasal augmentation approximately 3 years ago. The patient did not want to undergo surgical correction. Instead, she requested temporary treatment with a noninvasive procedure. To improve the drooping nose, strong rotation, projection, and augmentation were required. A few filler injection techniques that consider the characteristics of the dynamic structure of the nose for rotation, projection, and augmentation have been reported [3-8]. In this study, a modified filler injection technique that preferentially focused on the order of injection (tip rotation, projection, and then augmentation) was used.

\section{Case report}

\section{Development of a drooping nose following primary rhinoplasty}

For a simple dorsal augmentation of the nose, an I-shaped silicone implant was placed from the root to the supra-tip break through the marginal incision line of the right naris under local anesthesia (Fig. 1A, B). Postoperatively, the contour of the nasal dorsum was perfectly seen (Fig. 1B, C). However, after 3 months, the height of the root decreased and the volume of the supra-tip break increased (Fig. 1D). An obvious drooping nose with an unnatural lateral contour and a decrease in the nasolabial angle was clearly seen (Fig. 1E).

\section{Preoperative marking and anesthesia}

Local anesthesia ( $0.2 \%$ lidocaine solution) was injected into the entry sites. Preoperatively, the contour of the silicone implant and margin of the upper cartilage (ULC) were marked (Fig. 2).

\section{Sequential augmentation of filler injection technique}

The sequential augmentation of filler injection (SAFI) technique, which considers the dynamic structure of the nose, was used for rotation, projection, and augmentation. For tip rotation, $0.6 \mathrm{ml}$ of Elravie ${ }^{\circledR}$ Premier Ultravolume-L (hyaluronic acid 23 mg/ml, 0.3\% lidocaine; Humedix Co. Ltd., Anyang, Korea) was first injected into the space between the medial crus and anterior nasal spine $(0.4 \mathrm{ml})$; it was then injected into the intercolumellar space $(0.2 \mathrm{ml})$ via the entry site of the subnasale using a 23-gauge blunt cannula (Fig. 3A, B) [5,9]. For tip projection, a 23-gauge sharp needle was intranasally inserted into the perichondrium of the middle crus at the lower border of the LLC after retraction of the nostril using the first and second fingers. After aspiration, $0.1 \mathrm{ml}$ of filler was slowly injected into the perichondrium of the middle crus of the LLC, while the needle was drawn back (Fig. 3).

For dorsum augmentation, the filler was placed in the space between the distal margin of the contour of the descended silicone and supra-tip break point, and $0.4 \mathrm{ml}$ of filler was injected

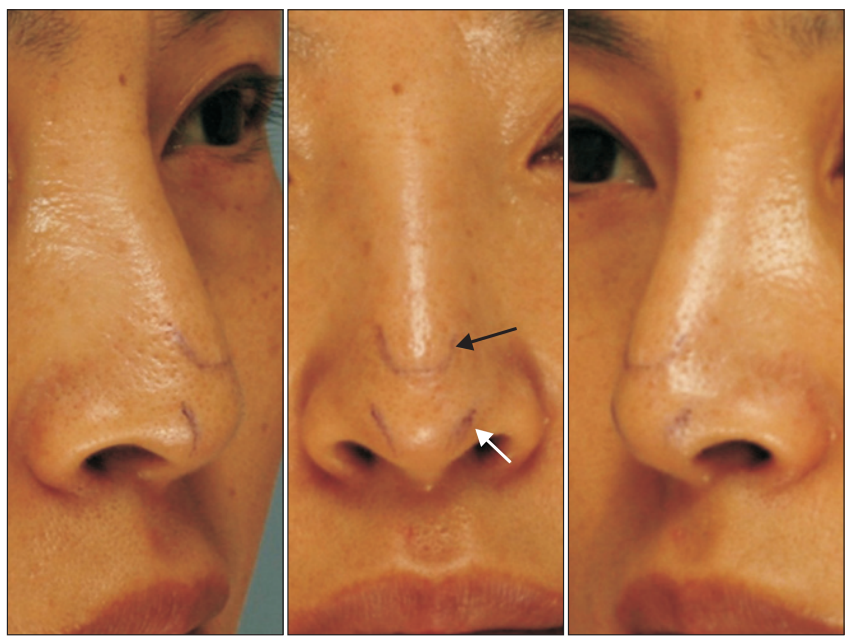

Fig. 2. Preoperative marking. The black arrow shows the lower margin of the silicone implant that descended, and the white arrow shows the lower lateral margin of the middle crus of the lower lateral cartilages.
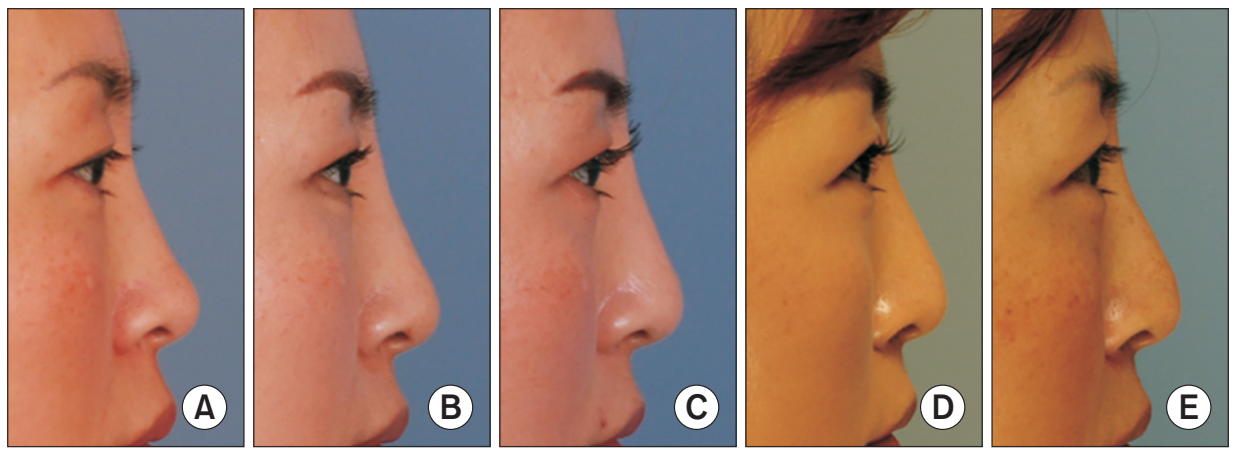

Fig. 1. Change in lateral contour after closed augmentation rhinoplasty using an I-shaped silicone implant. Postoperatively, the droopy nose is clearly visible (E). (A) Before, (B) 1 week after, (C) 6 weeks after, (D) 3 months after, and (E) 3 years after. 

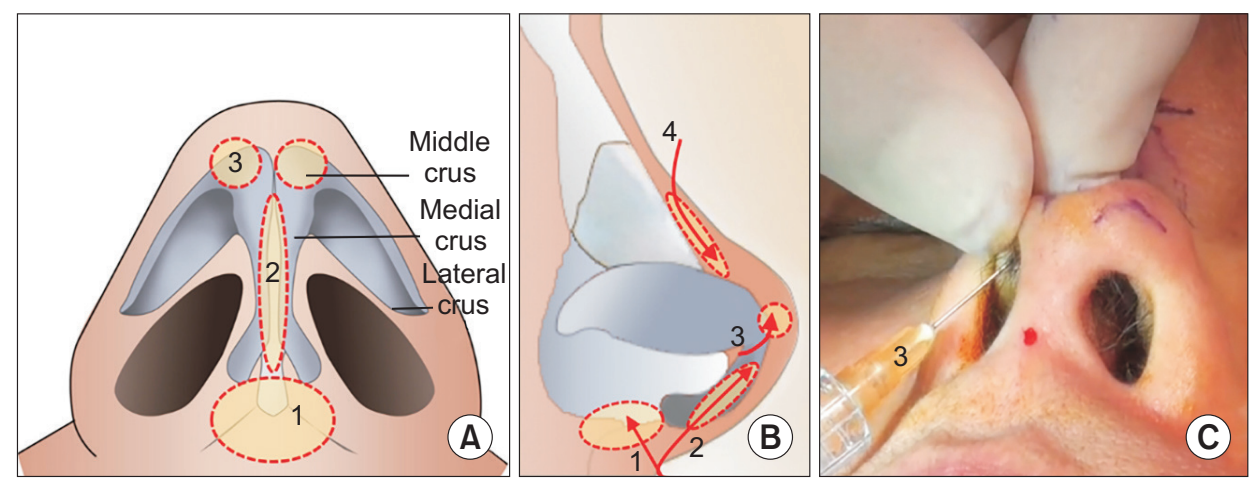

Fig. 3. Schematic basal view of the nose for the location and injection sequence of the filler (A). Schematic lateral view of the nose for the location and injection sequence of the filler (B), and a photo showing the intranasal approach for tip projection during procedure (3) (C). The filler was first injected into the space between the medial crus and anterior nasal spine (1) and then into the intercolumellar space (2) for tip rotation. Subsequently, it was injected intranasally into the perichondrium of the middle crus of the lower lateral cartilage for tip projection (3). Finally, it was injected into the perichondrium of the upper cartilage for tip augmentation (4). (A) Revised from the article of Moon HJ (Facial Plast Surg Clin North Am 2018;26:323-30) [5] with original copyright holder's permission. (B) Revised from Jung DH (Rhinoplasty; 2002. p. 12) [9] with the original copyright holder's permission.
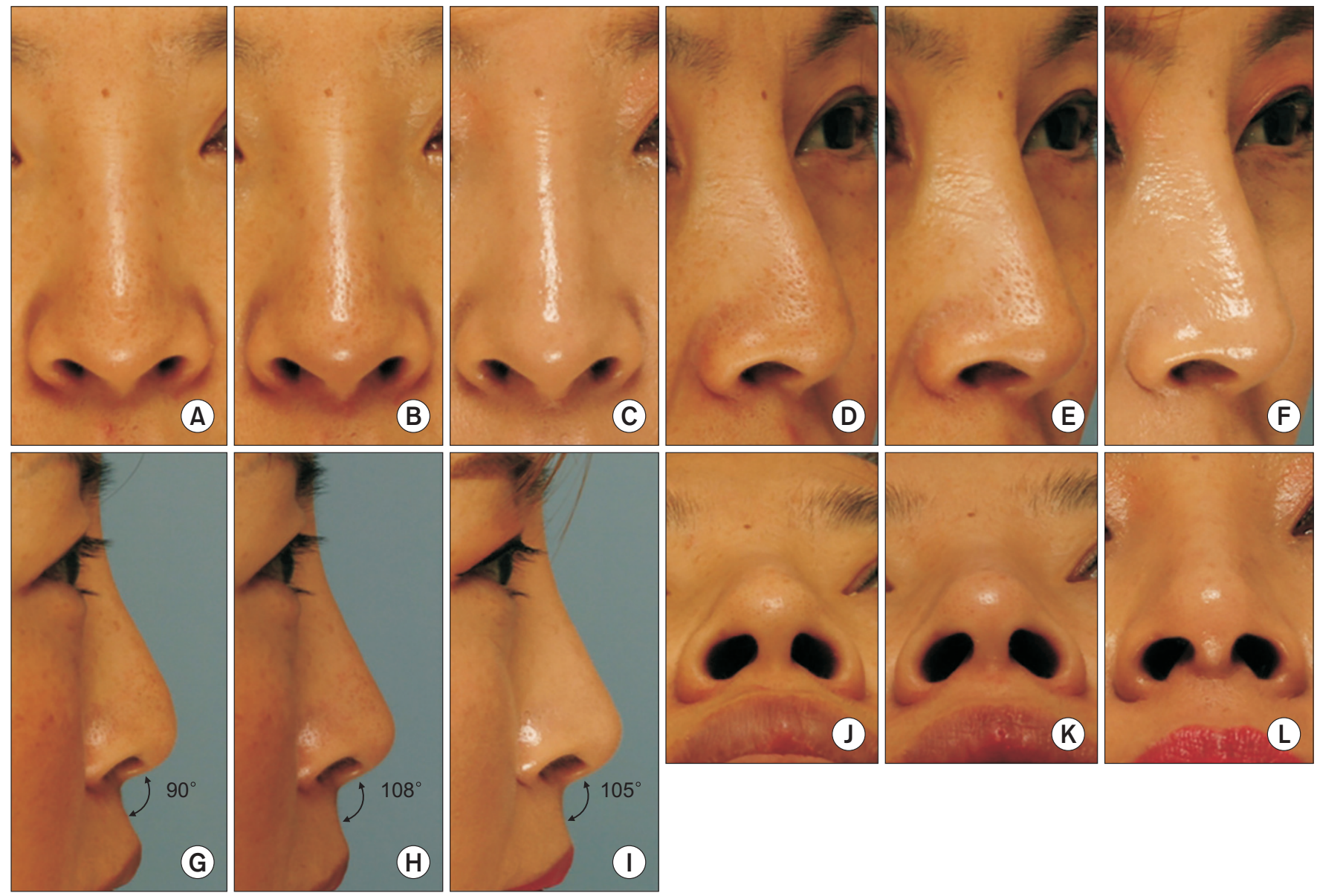

Fig. 4. A 42-year-old female had a filler injection $(1.9 \mathrm{ml})$ for the correction of a drooping nose caused by the descent of a silicone implant after augmentation rhinoplasty. (A, D, G, J) Before the procedure. (B, E, H, K) Two weeks after the procedure. (C, F, I, L) Four months after the procedure. The change of naso labialangles $(90,108$, and 105) are seen. 
into the perichondrium and into the deep fat layer along the middle of the ULC, while the needle was drawn back (Fig. 3B). After 2 weeks, through the subnasale, $0.3 \mathrm{ml}$ of filler was injected using a 23-gauge blunt cannula for further projection and rotation of the nose.

\section{Results}

The SAFI technique significantly improved the drooping nose, as shown in Fig. 4. The dorsal light reflex was extended to the supra-tip break point, and the straight line of the dorsal bridge was observed despite the volume increase in the nasal tip (Fig. 4B). The increased vertical length of the philtrum, less protruded upper lip, and increased length of the columella are depicted in Fig. 4B, D, and F. The nasolabial angle significantly increased from $90^{\circ}$ to $108^{\circ} 2$ weeks after the procedure to $105^{\circ}$ 4 months after the procedure (Fig. 4G-I). The tip projection was significantly increased (Fig. 4D-F). The nostril-to-infralobular ratio increased, and the shape of the nostril became more elliptical (Fig. 4J-L).

\section{Discussion}

Filler rhinoplasty has been commonly performed for nonsurgical nasal augmentation. To obtain a better outcome, some physicians have attempted to develop an advanced technique for rotation, projection, and augmentation that can prevent serious complications [3-8]. Moreover, two physicians have provided interesting opinions regarding the regular order of filler injection $[4,5]$. One of them suggested that the filler should be first injected into the subnasale for tip rotation and must then be injected from the radix to the tip with the sequence of tip rotation, dorsal augmentation, and tip projection in Western individuals [4]. Another physician suggested that the filler should be injected from the radix, rhinion, tip, to the supra-tip break point in Asian individuals with the sequence of dorsal augmentation, tip rotation, tip projection, and dorsal augmentation again [5]. In principle, I would agree with the first opinion because Western individuals have a high radix with strong and thick cartilage and with the second opinion in terms of the injection order from the tip to the supra-tip break point in Asian individuals. However, I do not agree with the injection technique for tip projection in which the filler is injected into the interdomal space [5] because it may lead to insufficient projection or, particularly, widening of the tip in Asian individuals as they have weak and thin cartilage. Considering the above-mentioned opinions, the filler should be injected in the order of tip rotation, tip projection, and then dorsal augmentation from the radix to the supra-tip break point. In addition, for definite and effective tip projection, the filler must be injected intranasally into the perichondrium of each of the LLC's middle crura.

In this case, the drooping nose caused by the descent of the silicone implant was corrected using the temporary expedient method using the SAFI technique. The outcome was believed to be achieved with the performance of tip projection after a strong rotation of the tip via volumetric support. Thus, a sufficient filler volume can be injected to cover the defective area between the distal contour of the silicone implant and nasal tip without a pollybeak deformity. The reduced nasolabial angle from $108^{\circ}$ to $105^{\circ}$ (Fig. $4 \mathrm{H}, \mathrm{I}$ ) and decreased tip projection (Fig. $4 \mathrm{E}, \mathrm{F}$ ) is indicative of the maturation of the wound healing process and settlement of the injected filler. It can be one of the factors that should be considered when predicting the optimal volume that will produce the greatest effect.

In conclusion, in the present case, a drooping nose caused by the descent of a silicone implant was temporarily corrected using the SAFI technique. Thus, the SAFI technique can be a standard and reliable procedure for filler rhinoplasty. However, further studies that include a high number of cases should be performed to validate the safety and efficacy of the procedure.

\section{Conflicts of interest}

The authors have nothing to disclose.

\section{References}

1. Sajjadian A, Guyuron B. An algorithm for treatment of the drooping nose. Aesthet Surg J 2009;29:199-206.

2. Lee KC, Kwon YS, Park JM, Kim SK, Park SH, Kim JH. Nasal tip plasty using various techniques in rhinoplasty. Aesthetic Plast Surg 2004;28:445-55.

3. Demir UL. Comparison of tongue-in-groove and columellar strut on rotation and projection in droopy nasal tip: contribution of a cap graft. J Craniofac Surg 2018;29:558-61.

4. Liew S, Scamp T, de Maio M, Halstead M, Johnston N, Silberberg $\mathrm{M}$, et al. Efficacy and safety of a hyaluronic acid filler to correct aesthetically detracting or deficient features of the Asian nose: a prospective, open-label, long-term study. Aesthet Surg J 2016;36:760-72.

5. Moon HJ. Injection rhinoplasty using filler. Facial Plast Surg Clin North Am 2018;26:323-30.

6. Johnson ON 3rd, Kontis TC. Nonsurgical rhinoplasty. Facial Plast Surg 2016;32:500-6.

7. Tanaka Y. Oriental nose occidentalization and perinasal 
shaping by augmentation of the underdeveloped anterior nasal spine. Plast Reconstr Surg Glob Open 2014;2:e197.

8. Jung DH, Tan KS, Jin SG. Nasal tip surgery: nasal lengthen- ing and augmentation in Asian rhinoplasty. J Cosmet Med 2017;1:8-15.

9. Jung DH. Rhinoplasty. Seoul: Greenbook Ltd.; 2002. p. 12. 